OPEN ACCESS

Edited by:

Qi Wang,

Southern Medical University, China

Reviewed by:

Keiichi Kadoyama,

Himeji Dokkyo University, Japan

Yingmin $L i$,

Hebei Medical University, China

*Correspondence:

Ruibin Su

ruibinsu@126.com

Luyang Tao

taoluyang@suda.edu.cn

Specialty section: This article was submitted to

Neuropharmacology,

a section of the journal

Frontiers in Neuroscience

Received: 18 November 2020 Accepted: 14 December 2020

Published: 14 January 2021

Citation:

Chen G, Wei X, Xu X, Yu G, Yong Z, Su R and Tao L (2021)

Methamphetamine Inhibits Long-Term Memory Acquisition and Synaptic Plasticity by Evoking Endoplasmic

Reticulum Stress.

Front. Neurosci. 14:630713. doi: 10.3389/fnins.2020.630713

\section{Methamphetamine Inhibits Long-Term Memory Acquisition and Synaptic Plasticity by Evoking Endoplasmic Reticulum Stress}

\author{
Guang Chen ${ }^{1}$, Xiaoning $\mathrm{Wei}^{2}$, Xiang $\mathrm{Xu}^{3}$, Gang $\mathrm{Yu}^{4}$, Zheng Yong ${ }^{4}$, Ruibin $\mathrm{Su}^{4 *}$ and \\ Luyang $\mathrm{TaO}^{1 *}$
}

${ }^{1}$ Department of Forensic Medicine, Medical School of Soochow University, Suzhou, China, ${ }^{2}$ Becton, Dickinson and Company, Guangzhou, China, ${ }^{3}$ School of Forensic Medicine, Wannan Medical College, Wuhu, China, ${ }^{4}$ State Key Laboratory of Toxicology and Medical Countermeasures, Beijing Institute of Pharmacology and Toxicology, Beijing, China

Methamphetamine (MA), an illicit drug abused worldwide, leads to cognitive impairment and memory loss. However, the detailed mechanisms of MA-induced neurologic impairment are still unclear. The present study aimed to investigate the mechanisms of MA-induced inhibition of memory acquisition from the perspective of endoplasmic reticulum (ER) stress. ER stress, caused by the accumulation of wrongly folded proteins in the ER, is important for new protein synthesis, which further influence the formation of long-term memory. A subacute MA poisoning model of mice was established and several behavioral experiments were performed, including elevated plus maze, Morris water maze, electro-stimulus Y-maze, and novel object recognition tasks. The present results suggested that 4 days exposure to MA induced significant memory loss. Whereas, this damage to memory formation could be protected when mice were pre-treated with ER stress inhibitor, tauroursodeoxycholic acid (TUDCA). The results of Western blotting showed that subacute exposure to MA increased the expression levels of ER stress marker proteins, such as binding immunoglobulin protein, phosphorylated eukaryotic translation initiation factor $2 \alpha$, cyclic AMP-dependent transcription factor (ATF)-4, ATF-6, and CCAAT-enhancer binding protein homologous protein. Meanwhile, the enhanced expression levels of these proteins were reversed by TUDCA, indicating that MA administration induced memory loss by evoking ER stress in the hippocampus. We also found that MA inhibited the induction of long-term potentiation (LTP) in the hippocampus. Nevertheless, LTP could be induced when mice were pre-treated with TUDCA. In conclusion, MA inhibited long-term memory acquisition and synaptic plasticity via ER stress.

Keywords: methamphetamine, neurotoxicity, endoplasmic reticulum stress, memory, tauroursodeoxycholic acid

\section{INTRODUCTION}

Methamphetamine (MA), an amphetamine-type stimulant, has been abused worldwide and its use has increased the heath and economic burden to individuals and society (Courtney and Ray, 2014; Xu et al., 2019). Acting mainly on the dopaminergic, noradrenergic, and serotonergic pathways of the central nervous system (Cruickshank and Dyer, 2009), MA induces significant 
neurotoxicity (Shaerzadeh et al., 2018), one of which is cognitive impairment. Clinical researchers have found that chronic MA abuse leads to cognitive dysfunction (Scott et al., 2007), which is related to attention, psychomotor speed, and executive function in addition to memory loss. It is also suggested by previous studies that MA-treated rodents exhibit deficits in spatial and recognition memory (Belcher et al., 2008; North et al., 2013). In contrast, MA-induced memory enhancement has also been detected in rodents (Cao et al., 2013). Collectively, the differing effects of MA on memory may be attributed to different models of MA administration and behavioral tasks carried out in different studies.

Oxidative stress is considered as one important mechanism of MA-induced neurotoxicity that causes nerve cell death by disrupting cellular organelle function, including that of the endoplasmic reticulum (ER) (Krasnova and Cadet, 2009; $\mathrm{Yu}$ et al., 2015). Induced by the accumulation of unfolded proteins and protein aggregation, ER stress is initiated by phosphorylated inositol requiring kinase $1 \alpha$ (p-IRE1 $\alpha)$, PKRlike ER kinase (PERK) or cyclic AMP-dependent transcription factor-6 (ATF-6). ER stress is mainly mediated by three pathways (Kim et al., 2008) and the role of ER stress is to reduce the protein load of the ER and promote the degradation or reassembly of misfolded proteins. However, if ER stress is too severe or lasts too long, it can lead to cell death (Sano and Reed, 2013). Recent studies have found that ER stress is involved in the pathophysiology of several diseases, including neurodegeneration, cancer, diabetes, stroke, and inflammation (Oyadomari and Mori, 2004). MA can also induce ER stress and lead to cell death via ER stress-associated autophagy and apoptosis (Jayanthi et al., 2004). However, it is still unclear whether ER stress is involved in MA-induced memory loss. As protein synthesis is essential for long-term memory induction, it can be postulated that ER stress may be involved in MA-induced long-term memory impairment.

In the present study, a subacute administration method of MA, which has been found to induce ER stress and neurotoxicity in mice (Cai et al., 2016), was used and different behavioral experiments, including elevated plus maze, Morris water maze, electro-stimulus Y-maze, and novel object recognition tasks, were conducted to investigate the impairment of memory caused by MA. Next, tauroursodeoxycholic acid (TUDCA), a general inhibitor for ER stress, was used to inhibit ER stress and the effect of TUDCA pre-treatment on MA-induced memory loss was evaluated. TUDCA, the taurine-conjugated derivative of ursodeoxycholic acid (UDCA) is a bile acid synthesized by intestinal bacteria, and used for the treatment of cholestatic liver diseases, diabetes, and atherosclerosis (Lebensztejn, 2000; Ozcan et al., 2006). Recent studies have found that TUDCA alleviates ER stress by enhancing ER folding ability and preventing protein aggregation (Liu et al., 2015). Meanwhile, we investigated the effect of ER stress on the synaptic plasticity by evaluating the role of ER stress in the induction of long-term potentiation (LTP) in the hippocampus, which is closely associated with memory formation and storage. LTP is defined as a long-term increase in synaptic response following a high frequency stimulation (HFS) (Baltaci et al., 2019). It has been suggested that LTP is not only a laboratory phenomenon, but also involved in the information storage (Pastalkova et al., 2006).

\section{MATERIALS AND METHODS}

\section{Drugs}

MA was obtained from the Beijing Institute of Pharmacology and Toxicology. TUDCA was purchased from Shanghai Aladdin Bio-Chem Technology Co., Ltd. (cat. no. S101371). All drugs were dissolved in the saline $(0.9 \% \mathrm{NaCl}$ solution) and prepared to a specified concentration. The mice were divided into four groups: (i) Saline, (ii) TUDCA, (iii) MA, and (iv) TUDCA + MA. In the MA group, mice were administered intraperitoneal (i.p.) injections of $15 \mathrm{mg} / \mathrm{kg}$ MA (twice a day) for 4 days. In the TUDCA + MA group, mice received i.p. injections of $200 \mathrm{mg} / \mathrm{kg}$ TUDCA $60 \mathrm{~min}$ before receiving MA injections every time. In the Saline and TUDCA groups, mice were administered i.p. injections of the saline and $200 \mathrm{mg} / \mathrm{kg}$ TUDCA, respectively.

\section{Animals and Housing}

Male C57BL/6 mice aged 6-8 weeks were obtained from SPF (Beijing) Biotechnology Co., Ltd. Mice had free access to water and food in standard experimental housing with a constant temperature range $\left(25^{\circ} \mathrm{C}\right)$ and a $12 \mathrm{~h}$ light/dark cycle. All experimental animal procedures were conducted in accordance with the National Institutes of Health (NIH) and were approved by the Animal Care and Use Committee of the Beijing Institute of Pharmacology and Toxicology. Different mice were used in each of the following experiments.

\section{Elevated Plus Maze}

Elevated plus maze (EPM) task was initially performed ( $n=8$ per group) to evaluate whether drug administration had an effect on animal anxiety-like behavior, using a standard experimental apparatus manufactured by Shanghai Jiliang Software Technology Co., Ltd. The apparatus consisted of two open arms $(30 \times 5 \mathrm{~cm}$, length $\times$ width $)$ and two closed arms $(30 \times 5 \times 15 \mathrm{~cm}$, length $\times$ width $\times$ height $)$ elevated $50 \mathrm{~cm}$ above the floor. The EPM testing was conducted as previously described (Psotta et al., 2015). At the beginning of the experiment, mice were placed in the central zone of the maze facing an open arm and were allowed to freely explore the maze for $5 \mathrm{~min}$. The maze was carefully cleaned after each trial to remove odor cues. EPM testing was performed $24 \mathrm{~h}$ after the final injection of drugs (Figure 1A). Increased anxiety-like behavior was indicated by a lower percent time in the open arms.

\section{Morris Water Maze}

The Morris water maze (MWM) task was performed ( $n=10$ per group) to evaluate the effect drug administration on the spatial memory formation of mice. The apparatus, which was manufactured by Shanghai Jiliang Software Technology Co., Ltd., consisted of a stainless-steel circular tank $(120 \mathrm{~cm}$ diameter, $60 \mathrm{~cm}$ height) filled with $23 \pm 1^{\circ} \mathrm{C}$ water to a depth of $40 \mathrm{~cm}$. A Plexiglas platform (10 cm diameter) was submerged $1.5 \mathrm{~cm}$ below the surface of the water during the training session and 


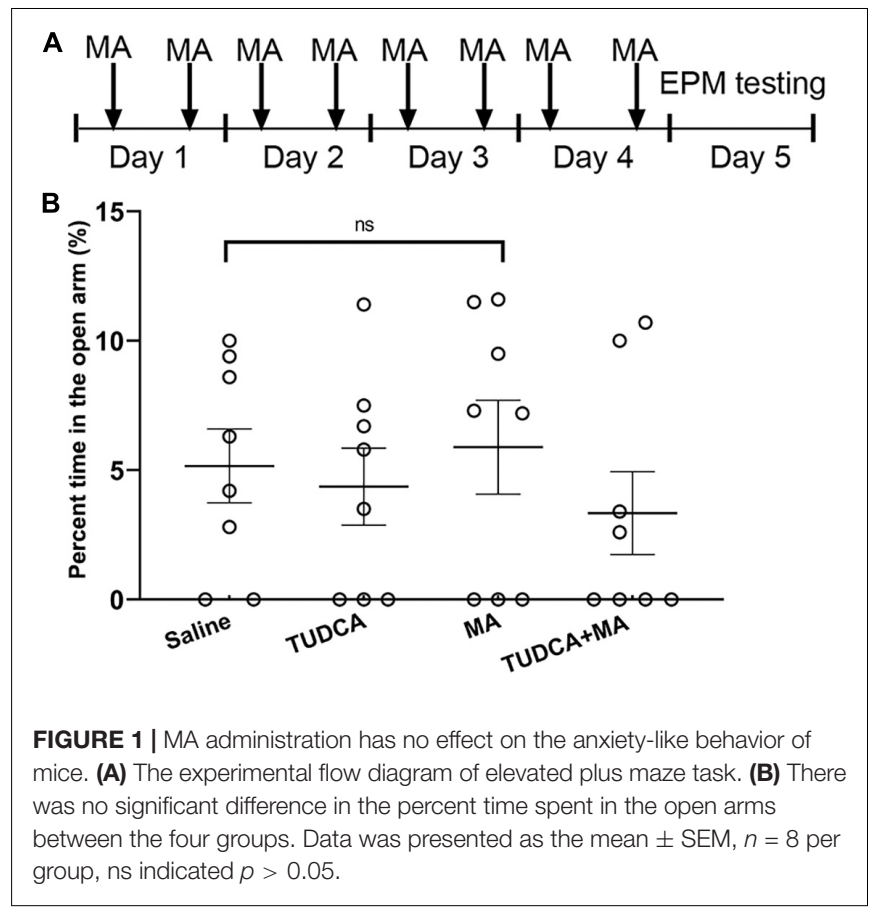

distal cues were placed within the experimental room. The protocol of MWM task was according to the previous reports (Vorhees and Williams, 2006; Cao et al., 2013) with some modifications. Mice were administered i.p. injections of drugs for 4 days followed by 4 days training with testing conducted $24 \mathrm{~h}$ after the last training session (Figure 2A). During the training session, mice were held facing the tank wall and randomly placed into the pool from one of four fixed entry points and allowed to swim freely for $90 \mathrm{~s}$. A trial ended when the mouse climbed onto the platform and stopped for $5 \mathrm{~s}$ or when the $90 \mathrm{~s}$ time limit had elapsed. Mice were given a 15 min rest between two consecutive trials. A $60 \mathrm{~s}$ probe testing was performed $24 \mathrm{~h}$ after the last day of training with the platform removed. Swimming tracks were recorded and analyzed. Escaped latency (i.e., the interval between the start of the experiment and when mice climbed onto the platform) was used to evaluate spatial learning during the training stage. During the testing, platform site crossings and crossing latency (i.e., the interval between the start of the test and when mice swam across the site of the platform) were used to evaluate spatial memory retrieval.

\section{Electro-Stimulus Y-Maze}

Electro-stimulus Y-maze task was performed ( $n=8$ per group) to evaluate the effect of drug administration on the formation of recognition memory. The apparatus was manufactured by Zhangjiagang Biomedical Equipment Manufacturing Co., Ltd, and consisted of 3 arms with equal dimensions $(65 \times 15 \times$ $15 \mathrm{~cm}$, length $\times$ width $\times$ height), two of which were arranged at $120^{\circ}$ angles. At the bottom of each arm, there were conductive gratings that were $0.5 \mathrm{~cm}$ in diameter and $0.5 \mathrm{~cm}$ in space. Each arm contained a light source. Three seconds after a light was turned on in one arm, an electrical stimulation lasting 10 $s$ was given in the other two arms as well as the junctional zone. The task was performed as previously reported (Yu et al., 2003) with the procedure modified according to our experimental design. Mice were initially administered i.p. injections of drugs for 4 days, followed by 4 days training with 20 trials each day (Figure 3A). When performing the trials, electrical stimulation was given at random in the three arms and the junctional zone to help mice learn and remember that the arm in which the light was on was a safe area. If a mouse escaped into the safe arm within $10 \mathrm{~s}$ following electrical stimulation, the response would be recognized as correct. The testing, which consisted of 20 trials of electrical stimulation, was performed $24 \mathrm{~h}$ after the 4 days of training. For each mouse, 18 or more correct responses within 20 trials indicated learnt. Indices to evaluate the recognition memory included the percentages of correct trials and mice exhibiting learnt.

\section{Novel Object Recognition}

Novel object recognition (NOR) task was performed using a Plexiglas box $(40 \times 40 \times 40 \mathrm{~cm}$, length $\times$ width $\times$ height $)$, manufactured by Shanghai Jiliang Software Technology Co., Ltd. The task consisted of three processes: habituation, training and testing. During the habituation (day 5), there was no object in the arena and mice were allowed to move freely for $20 \mathrm{~min}$ to become familiar with the environment. During the training (day 6), two identical objects were placed in the arena and mice were allowed to explore the objects for $10 \mathrm{~min}$. During the testing, one of the objects was replaced with a novel object and mice were placed in the arena for $7 \mathrm{~min}$ to explore the two objects. In the present study, Test 1 and Test 2 were performed $30 \mathrm{~min}$ and 24 h post the training, respectively (Figure 4A). Novel object index (NOI), which is the time spent exploring the novel object divided by the time spent exploring the two objects, was used to evaluate the formation of recognition memory.

\section{Western Blotting}

Mice were killed by an overdose of isoflurane $24 \mathrm{~h}$ after the last ingestion of MA and the hippocampal tissue was dissected on ice to extract the whole protein fraction. Western blotting was performed as previously reported (Cai et al., 2016) to examine the expression of ER stress marker proteins, including binding immunoglobulin protein (Bip) (Abcam, ab21685), cyclic AMPdependent transcription factor (ATF)-4 (Abclonal, A8687), phosphorylated eukaryotic translation initiation factor $2 \alpha$ (peIF2 $\alpha$ ) (CST, 3398s), ATF-6 (Abclonal, A0202) and CCAATenhancer binding protein homologous protein (Chop) (Abclonal, A0221). Values of these proteins were normalized to that of actin (Applygen, C1313). Bands were semi-quantified using Image J software (NIH).

\section{Electrophysiology}

The effect of MA on the induction of LTP in vivo of the perforant path (PP)-dentate gyrus (DG) pathway in the hippocampus was investigated as previously described (Gureviciene et al., 2004). Mice ( $n=5$ per group) were anesthetized with urethane $(1.5 \mathrm{~g} / \mathrm{kg}$, i.p.) and then a pair of stimulating electrodes were implanted into the perforant path of the left hemisphere at A/P: $-3.8 \mathrm{~mm}$, 


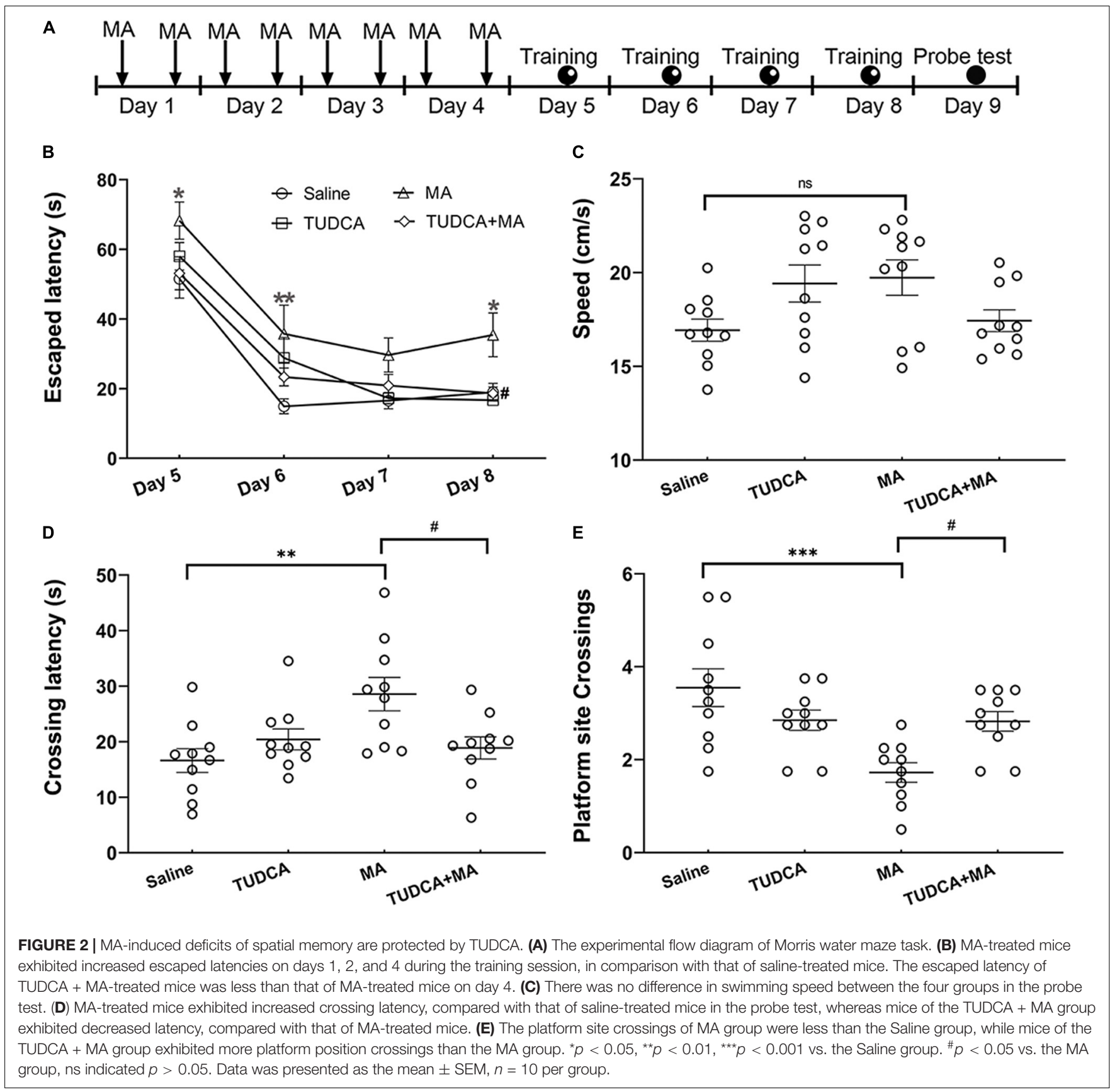

M/L: $-3.0 \mathrm{~mm}, \mathrm{D} / \mathrm{V}:-1.5 \mathrm{~mm}$ (from the dura). Meanwhile, a pair of recording electrodes were implanted into the dentate gyrus of the left hemisphere at A/P: $-2.0 \mathrm{~mm}, \mathrm{M} / \mathrm{L}:-1.4 \mathrm{~mm}$, $\mathrm{D} / \mathrm{V}:-1.5 \mathrm{~mm}$ (from the dura, Figure 6A). The population spike (PS) was induced using monopolar pulses (duration, $400 \mu \mathrm{s}$; frequency, $1 / 30 \mathrm{~Hz}$ ) using an Isolated Pulse Stimulator (A-M SYSTEMS Co., Ltd.) The PS was reported using a Differential AC Amplifier (A-M SYSTEMS Co., Ltd.) and Axon Digidata 1550A Data Acquisition System (Molecular Devices Co., Ltd.). When the stabilized PS (Figure 6B) lasted at least $30 \mathrm{~min}$, we regulated the stimulating current to yield a PS that was $30-50 \%$ of the maximum amplitude. The PS was recorded for $30 \mathrm{~min}$ and the amplitude of PS was homogenized as the baseline. Then the HFS, consisting of three trains of 10 bursts (duration, 400 $\mu \mathrm{s}$; frequency, $300 \mathrm{~Hz}$ ) with an interval of $10 \mathrm{~s}$ between each train, was used to induce LTP in vivo. Next, the PS was yielded using formerly single monopolar pulses and recorded for $60 \mathrm{~min}$. The amplitude of PS was calculated using pClamp10.0 software (Molecular Devices Co., Ltd.; Figure 6C).

\section{Statistical Analysis}

All experiments were randomized and performed in a blinded manner. Data was presented as the mean \pm SEM. Statistical analysis was performed using GraphPad Prism 8.0 (GraphPad 
A

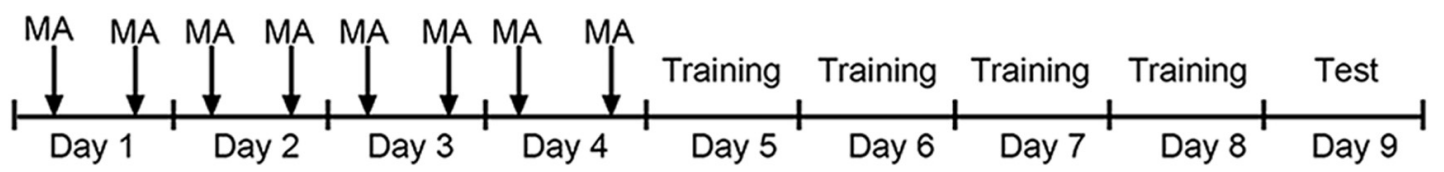

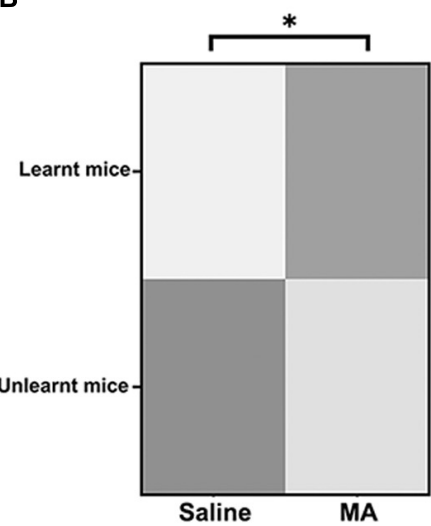

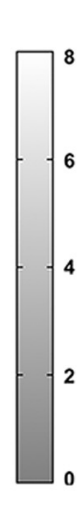

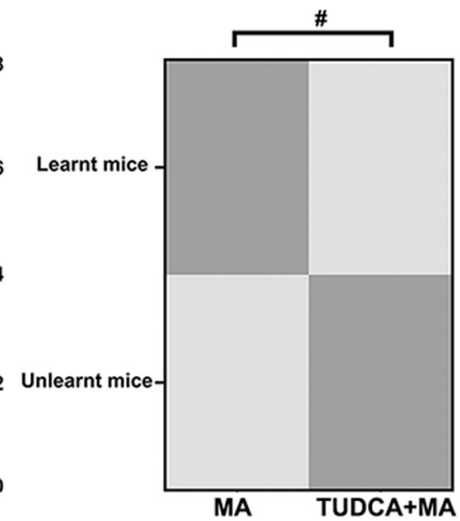
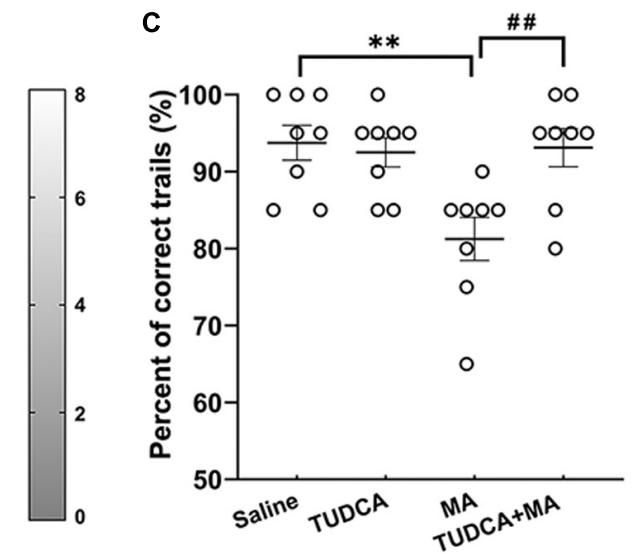

FIGURE 3 | MA-induced impairment of recognition memory is protected by TUDCA in electro-stimulus Y-maze. (A) The experimental flow diagram of electro-stimulus Y-maze task. (B) The number of mice exhibiting learnt and unlearnt in different groups. The present results indicated that a lower percent of MA-treated mice exhibited learnt in comparison with the saline group, whereas the percentage of mice exhibiting learnt in the TUDCA + MA group was higher than that of the MA group. (C) The percentage of correct trials in MA group was lower than that of the Saline group, while mice of the TUDCA + MA group represented a higher percent of correct trials in comparison with the MA group. ${ }^{*} p<0.05,{ }^{* *} p<0.01$ vs. the Saline group. ${ }^{\#} p<0.05,{ }^{\# \#} p<0.01$ vs. the MA group. Data was presented as the mean \pm SEM, $n=8$ per group.
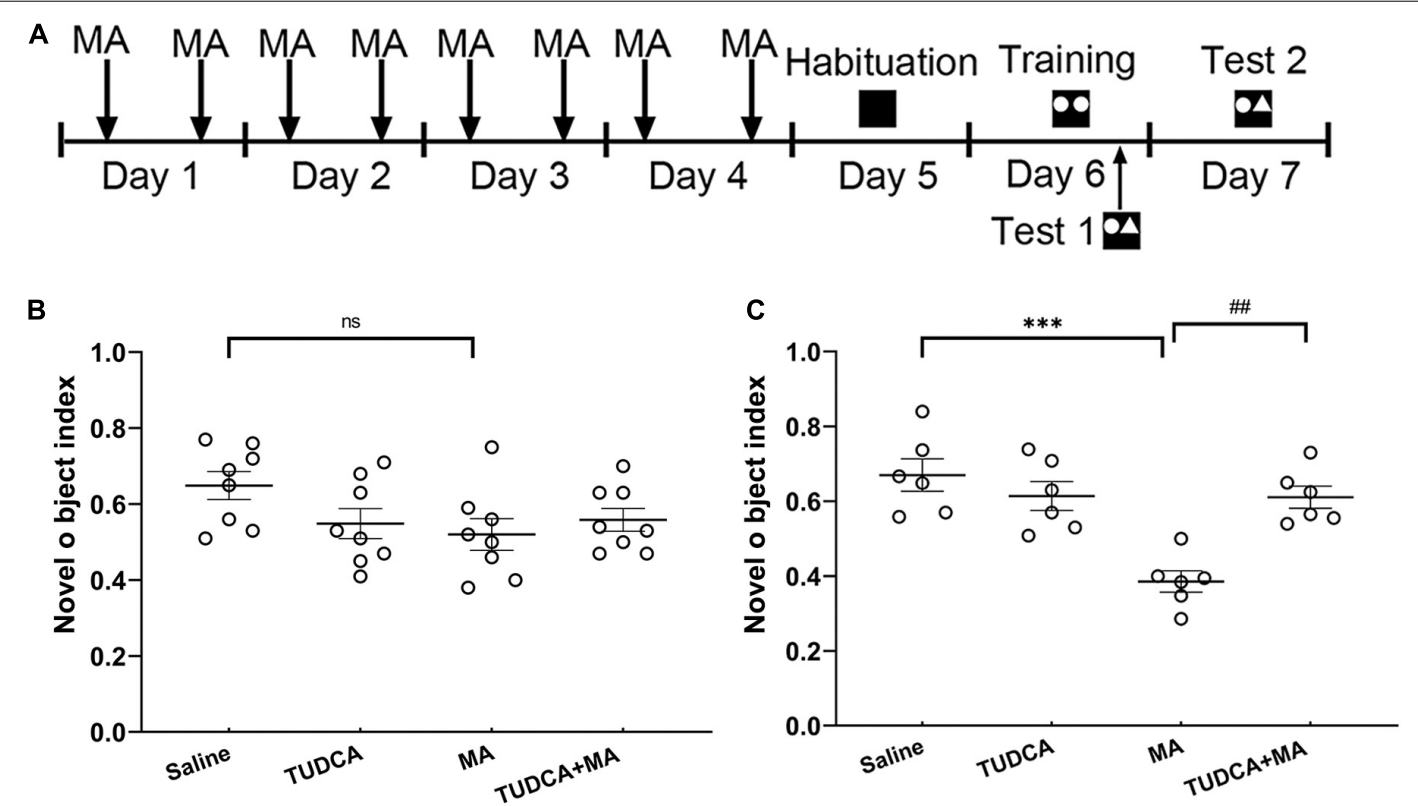

FIGURE 4 | MA-induced long-term recognition memory loss in novel object recognition task is avoided by TUDCA. (A) The experimental flow diagram of NOR task. (B) In Test 1, there was no difference in the novel object index (NOI) between the four groups. (C) In Test 2, MA-treated mice exhibited a lower NOI, compared with that of the Saline group, TUDCA + MA-treated mice exhibited a higher NOI in comparison with that of the MA group. ${ }^{* \star *} p<0.001$ vs. the Saline group. ${ }^{\# \#} p<0.01$ vs. the MA group, ns indicated $p>0.05$. Data was expressed as the mean $\pm \mathrm{SEM}, n=8$ per group.

Software, Inc.). For EPM testing, a one-way ANOVA was used to analyze the difference in the percent time spent in the open arms. For MWM testing, a two-way repeated-measures ANOVA followed by Bonferroni's multiple comparison test was used to analyze the difference in escaped latency and a one-way ANOVA followed by Bonferroni's multiple comparison test was used 
to analyze the difference in crossing latency and platform site crossings. For electro-stimulus Y-maze testing, a chi-square test was used to analyze the difference in the percentage of mice exhibiting learnt; a one-way ANOVA followed by Bonferroni's multiple comparison test was used to analyze the percentage of correct trials. For NOR testing, a one-way ANOVA followed by Bonferroni's multiple comparison test was used to analyze the difference in NOI. For electrophysiological experiments, a paired $t$-test was used to determine the difference in PS amplitude before and post HFS between different groups. The level of statistical significance was set at $p<0.05$.

\section{RESULTS}

\section{MA Administration Has No Effect on the Anxiety-Like Behavior of Mice}

The present results showed that there was no significant difference $(p>0.05)$ in the percent time spent in the open arms between the four groups (Figure 1B). Therefore, 4 days i.p. injections of MA had no effect on the tension and anxiety of mice.

\section{TUDCA Pre-treatment Ameliorates the Impairment of Spatial Memory Caused by MA}

In the MWM testing, a two-way repeated-measures ANOVA of escaped latency revealed significant effects of time $\left[F_{(3,36)}=74.38, p<0.001\right]$ and groups $\left[F_{(3,36)}=7.848\right.$, $p<0.001]$, but not their interaction $\left[F_{(9,108)}=0.684, p>0.05\right]$. MA-treated mice exhibited increased escaped latencies on day 1 $(p<0.05)$, day $2(p<0.01)$, and day $4(p<0.05)$ of the training session, compared with that of saline-treated mice; however, there was no difference in the escaped latency between the TUDCA + MA and Saline groups. Moreover, the escaped latency of TUDCA + MA-treated mice was less than that of MA group on the fourth day of training ( $p<0.05$; Figure 2B). In the probe test, MA-treated mice exhibited an increased crossing latency $(p<0.01)$ and decreased platform site crossings $(p<0.001)$ in comparison with saline-treated mice, whereas mice of the TUDCA + MA group exhibited a decreased crossing latency $(p<0.05)$ and increased platform site crossings $(p<0.05)$, compared with MA-treated mice (Figures 2D,E). Meanwhile, no difference in the swimming speed was found between the four groups $(p>0.05)$ in the probe test (Figure 2C).

\section{MA-Induced Recognition Memory Defects Are Rescued by TUDCA Pre-treatment}

In the electro-stimulus Y-maze testing, it was found that the percentage of mice exhibiting learnt in the MA group was lower than that of the Saline group $(p<0.05)$, meanwhile, the percentage of correct trials in the MA group was lower than that of the Saline group $(p<0.01)$. An increased percentage of TUDCA + MA-treated mice exhibited learnt $(p<0.05)$, compared with the MA group. The percentage of correct trials in the TUDCA + MA group was also higher than that of the MA group $(p<0.01$; Figures 3B,C). In the NOR testing, no significant difference in NOI was found between the four groups in Test 1 $(p>0.05$; Figure 4B). However, in Test 2, the NOI of the MA group was lower than that of the Saline group $(p<0.001)$. Mice of the TUDCA + MA group exhibited an increased NOI, compared with the MA group ( $p<0.01$; Figure 4C).

\section{MA-Induced ER Stress Is Inhibited by TUDCA Pre-treatment}

The results of the Western blotting suggested that the administration of MA in the present study increased the expression levels of ER stress marker proteins, including Bip ( $p$ $<0.01)$, ATF-6 $(p<0.05)$, ATF-4 $(p<0.01), p$-eIF $2 \alpha(p<0.05)$, and Chop $(p<0.01)$, all of which were reversed by TUDCA (Figures 5A,B).

\section{MA-Induced Inhibition of LTP in vivo Is Reversed by TUDCA Pre-treatment}

The obtained data indicated that the HFS used in the present study induced LTP in vivo in the hippocampus, evidenced by the PS amplitude of saline-treated mice was enhanced to $132.00 \pm 3.43 \%$ of the baseline $(p<0.001)$ post-HFS (Figures 6D,E). After the mice were administered i.p. injections of MA, however, the amplitude of PS could not be enhanced by the HFS $(p>0.05$; Figures 6F,G). When mice were pre-treated with TUDCA, the PS amplitude was increased to $143.30 \pm 6.56 \%$ of the baseline $(p<0.01)$ (Figures 6H,I).

\section{DISCUSSION}

MA is a worldwide abused illicit drug (Centazzo et al., 2019; $\mathrm{Xu}$ and Liu, 2019) and MA-induced cognitive impairment has been of increasing concern to the public (Morgan et al., 2012). Recently, several potential mechanisms underlying MA-induced neural damage have been proposed, including hyperthermia, excitotoxicity, inflammation, mitochondrial dysfunction, and oxidative stress (Yamamoto et al., 2010; Moszczynska and Callan, 2017). ER stress, which is involved in a variety of diseases, has been given much attention and considered as one mechanism mediating MA-induced neurotoxicity. Nevertheless, studies regarding the association between ER stress and MAinduced neural damage have largely focused on molecular mechanisms rather than behavioral manifestation. According to the molecular toxicology studies in our laboratory and other laboratories, it is postulated that ER stress may be one mechanism underlying the cognitive dysfunction caused by MA. To verify this hypothesis, the influence of MA administration on the memory formation was studied using different behavioral tests, including the MWM, electro-stimulus Y-maze, and NOR tasks. EPM testing was first performed to evaluate the effect of MA on anxiety-like behavior in mice. Compared with salinetreated mice, mice administered i.p. injections of MA exhibited no difference in the percentage of time spent in open arms, indicating that MA ingestion did not affect anxiety-like behavior of mice. MWM task was performed to investigate whether ER stress was involved in MA-induced spatial memory impairment. 


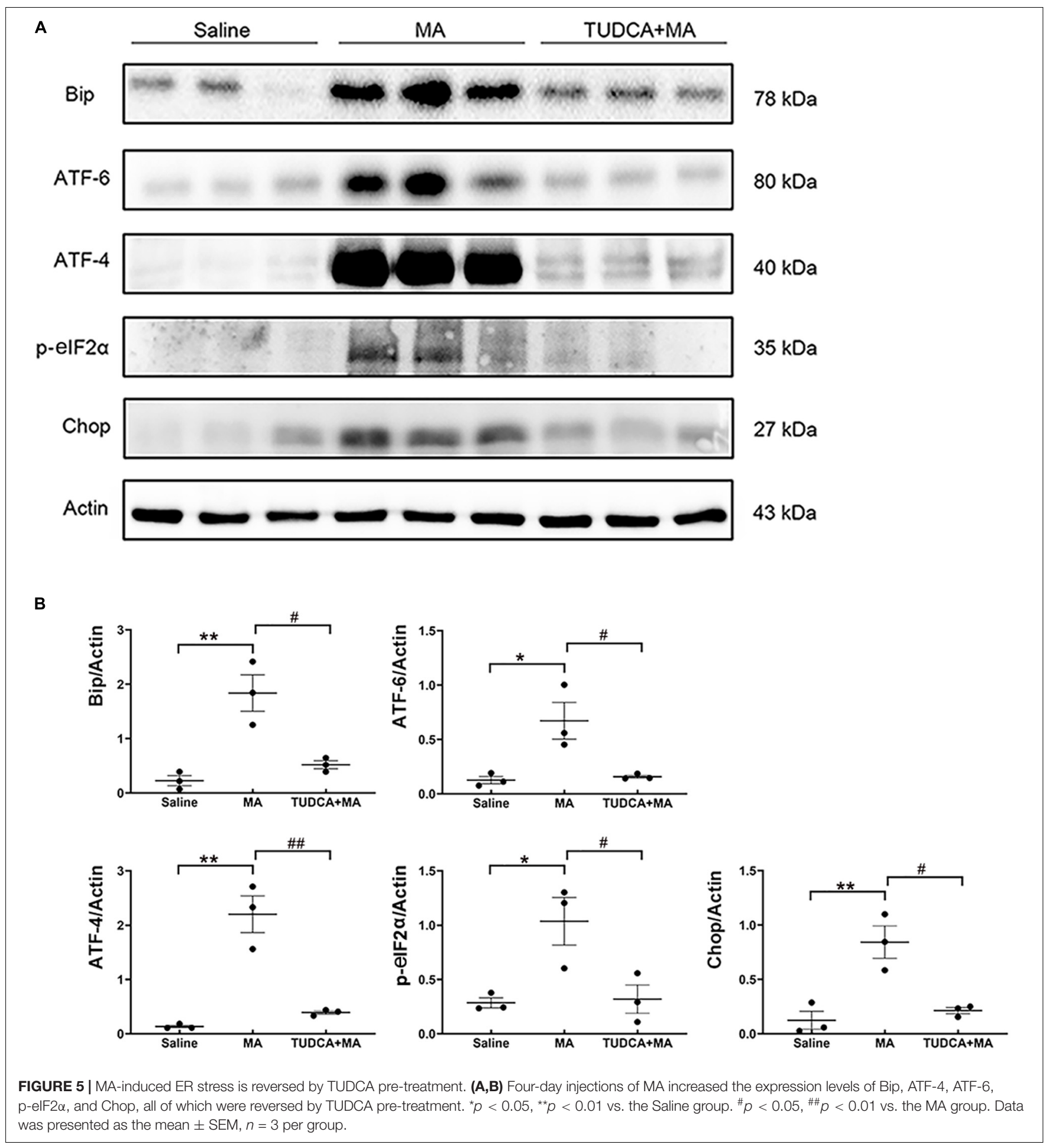

Consistent with the findings of previous reports (Vorhees and Williams, 2006; Heysieattalab et al., 2016), MA induced spatial memory impairment, as indicated by the increased escaped latencies during the training stage, crossing latency as well as fewer platform site crossings in the probe test. Compared with MA-treated mice, TUDCA + MA-treated mice exhibited decreased escaped latency, crossing latency as well as more platform site crossings, demonstrating that inhibiting ER stress rescued MA-induced impairment of spatial memory. Besides spatial memory, we also evaluated the effect of MA exposure on recognition memory by conducting electro-stimulus Y-maze and NOR tasks. The present results of these two experiments suggested that MA-induced inhibition of recognition memory could also be protected by TUDCA pre-treatment. Meanwhile, 
A

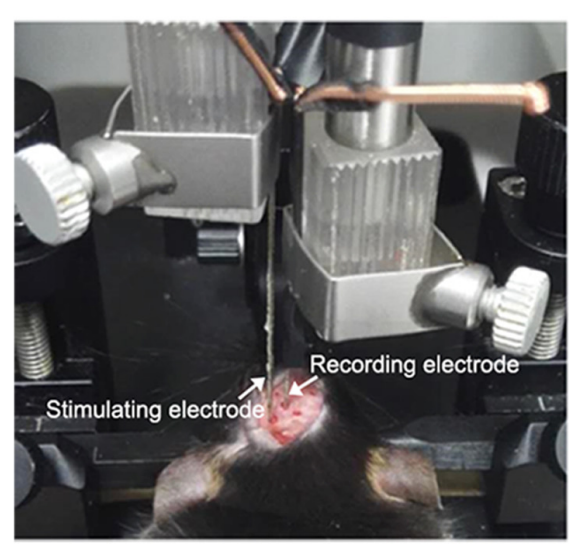

D
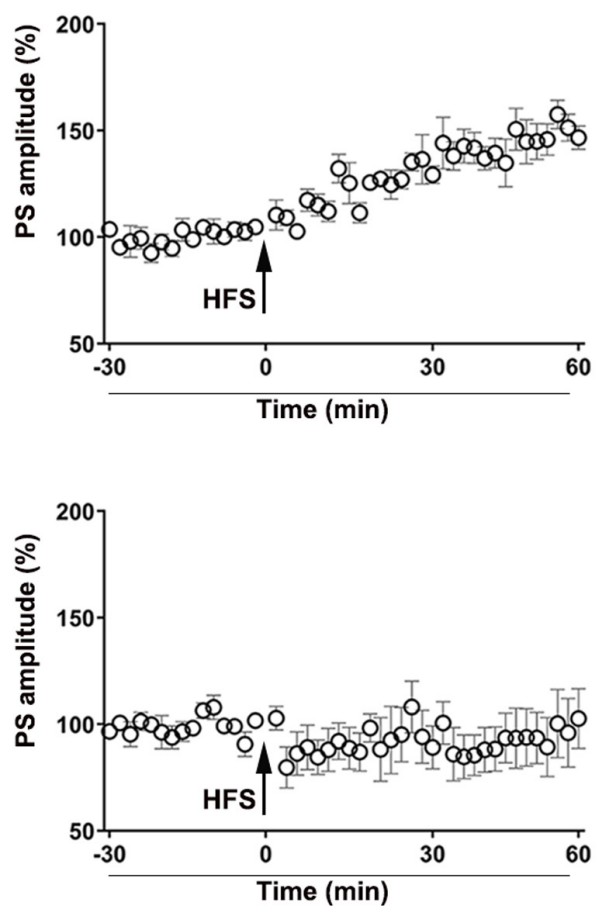

H

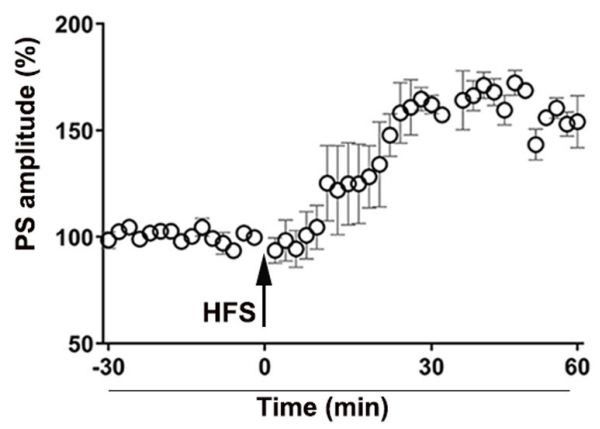

B

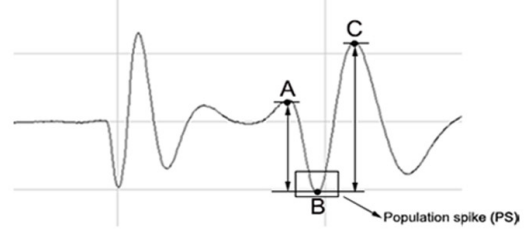

C

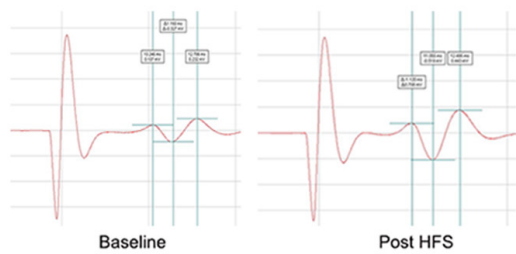

E

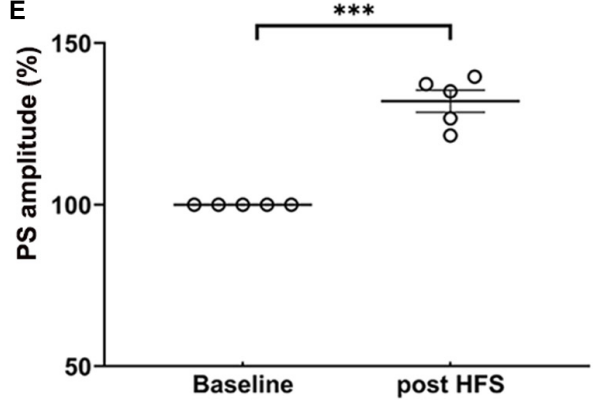

G

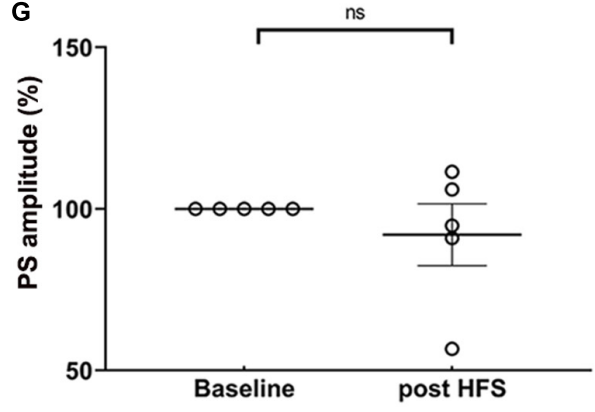

I

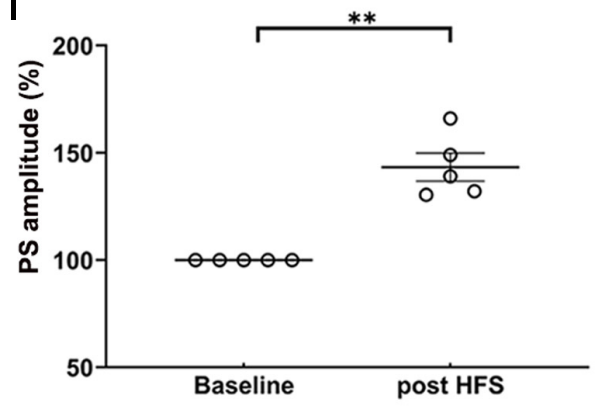

FIGURE 6 | MA-induced inhibition of LTP in vivo is reversed by TUDCA pre-treatment. (A) The position of stimulating electrodes and recording electrodes in the electrophysiological experiments. (B) A typical form of the population spike (PS) was consisted of a descending branch (AB) and an ascending branch (BC). The amplitude of PS was calculated by the average of the potential difference of AB and BC. (C) A typical enhancement of PS amplitude post-HFS, compared with the baseline. (D,E) The HFS induced a significant increase of the PS amplitude in the Saline group. $(\mathbf{F}, \mathbf{G})$ When mice were treated with MA, there was no difference in the PS amplitude post-HFS in comparison with the baseline. (H,I) When mice were pretreated with TUDCA, the HFS induced a prominent enhancement of the PS amplitude. ${ }^{* *} p<0.01,{ }^{* \star *} p<0.001$ vs. the baseline, ns indicated $p>0.05$. Data was presented as the mean $\pm \mathrm{SEM}, n=5$ per group. 
we evaluated the effect of MA administration on the expression levels of ER stress marker proteins in the hippocampus, such as Bip, ATF-6, ATF-4, p-eIF2 $\alpha$, and Chop. The results showed that MA increased the expression levels of these proteins, while TUDCA pre-treatment reversed the effect of MA, confirming that the subacute administration of MA induced cognitive impairment via ER stress in the present study.

In the NOR tasks, two different tests were conducted to distinguish the effect of MA ingestion on working memory and long-term memory. In Test 1, MA-treated mice did not show a decreased NOI, while MA-treated mice had a lower NOI in Test 2, indicating 4 days injections of MA impaired long-term recognition memory, but had no effect on short-term recognition memory. It has been generally accepted that memory can be divided into two different forms, short-term memory (STM) and long-term memory (LTM) (Scoville and Milner, 1957), which have different duration as well as molecular mechanisms. Previous studies have suggested that newly synthesized proteins are essential for the formation of LTM rather than STM (Davis and Squire, 1984). Recently, researchers have further found that increased expression level of p-eIF2 $\alpha$ impairs the induction of long-lasting LTP (L-LTP) and consolidation of spatial memory, indicating that translational control of gene expression by eIF $2 \alpha$ signaling pathway may be a molecular switch for LTM formation (Costa-Mattioli and Sonenberg, 2008). Enhanced phosphorylation of eIF $2 \alpha$ serves a negative control of the gene expression and protein synthesis (Dever, 2002) and p-eIF $2 \alpha$ is also involved in the ER stress. In the present study, MA administration increased the phosphorylation of eIF $2 \alpha$ and the expression level of ATF-4, which is a downstream of p-eIF $2 \alpha$. It was speculated that the inhibition of gene translation caused by $\mathrm{p}$-eIF $2 \alpha$ may be the reason why MA ingestion disturbed the formation of LTM, but not STM.

At last, the effect of MA poisoning on synaptic plasticity in the PP-DG pathway of the hippocampus was investigated to study the underlying mechanisms for MA-induced memory loss. We selected LTP in vivo, an intensively studied cellular model of the memory, to further study the role of MAinduced ER stress in the disruption of memory acquisition. LTP, referring to a sustained increase in efficiency of synaptic

\section{REFERENCES}

Baltaci, S., Mogulkoc, R., and Baltaci, A. (2019). Molecular Mechanisms of Early and Late LTP. Neurochem. Res. 44, 281-296. doi: 10.1007/s11064-018-2695-4

Belcher, A., Feinstein, E., O'dell, S., and Marshall, J. (2008). Methamphetamine influences on recognition memory: comparison of escalating and single-day dosing regimens. Neuropsychopharmacology 33, 1453-1463. doi: 10.1038/sj. npp. 1301510

Bliss, T., and Lomo, T. (1973). Long-lasting potentiation of synaptic transmission in the dentate area of the anaesthetized rabbit following stimulation of the perforant path. J. Physiol. 232, 331-356. doi: 10.1113/jphysiol.1973.sp010273

Cai, D., Huang, E., Luo, B., Yang, Y., Zhang, F., Liu, C., et al. (2016). Nupr1/Chop signal axis is involved in mitochondrion-related endothelial cell apoptosis induced by methamphetamine. Cell Death Dis. 7:e2161. doi: 10.1038/cddis. 2016.67

Cao, G., Zhu, J., Zhong, Q., Shi, C., Dang, Y., Han, W., et al. (2013). Distinct roles of methamphetamine in modulating spatial memory consolidation, retrieval, reconsolidation and the accompanying changes of ERK and CREB activation transmission caused by trains of high-frequency stimulation, was first fully described by Bliss and Lomo (1973). A great deal of research has revealed that LTP may be a biological substrate for at least some forms of memory (Lynch, 2004). In the present study, we found that MA-induced inhibitory of LTP in the hippocampus could also be reversed by ER stress inhibitor, TUDCA, indicating that ER stress may be a reason why LTP induction was inhibited by MA ingestion. However, the detailed mechanism for MA restraining LTP in vivo through ER stress needs further investigation in the future studies. In conclusion, MA inhibited long-term memory acquisition and synaptic plasticity by evoking ER stress.

\section{DATA AVAILABILITY STATEMENT}

The raw data supporting the conclusions of this article will be made available by the authors, without undue reservation, to any qualified researcher.

\section{ETHICS STATEMENT}

The animal study was reviewed and approved by the Animal Care and Use Committee of the Beijing Institute of Pharmacology and Toxicology.

\section{AUTHOR CONTRIBUTIONS}

GC conducted all of the experiments with the help of GY and ZY and wrote the manuscript with the help of XW. LT and RS designed the research. XX analyzed the results. All authors contributed to the article and approved the submitted version.

\section{ACKNOWLEDGMENTS}

We thank all the members of our laboratory for their helpful assistance.

in hippocampus and prefrontal cortex. Neuropharmacology 67, 144-154. doi: 10.1016/j.neuropharm.2012.10.020

Centazzo, N., Frederick, B., Jacox, A., Cheng, S., and Concheiro-Guisan, M. (2019). Wastewater analysis for nicotine, cocaine, amphetamines, opioids and cannabis in New York City. Forensic. Sci. Res. 4, 152-167. doi: 10.1080/20961790.2019. 1609388

Costa-Mattioli, M., and Sonenberg, N. (2008). Translational control of gene expression: a molecular switch for memory storage. Progr. Brain Res. 169, 81-95. doi: 10.1016/s0079-6123(07)00005-2

Courtney, K. E., and Ray, L. A. (2014). Methamphetamine: an update on epidemiology, pharmacology, clinical phenomenology, and treatment literature. Drug Alcohol Depend 143, 11-21. doi: 10.1016/j.drugalcdep.2014. 08.003

Cruickshank, C., and Dyer, K. (2009). A review of the clinical pharmacology of methamphetamine. Addiction 104, 1085-1099. doi: 10.1111/j.1360-0443.2009. 02564.x

Davis, H. P., and Squire, L. R. (1984). Protein synthesis and memory: a review. Psychol. Bull. 96, 518-559. doi: 10.1037/0033-2909.96.3.518 
Dever, T. E. (2002). Gene-specific regulation by general translation factors. Cell 108, 545-556. doi: 10.1016/s0092-8674(02)00642-6

Gureviciene, I., Ikonen, S., Gurevicius, K., Sarkaki, A., Van Groen, T., Pussinen, R., et al. (2004). Normal induction but accelerated decay of LTP in APP + PS1 transgenic mice. Neurobiol. Dis. 15, 188-195. doi: 10.1016/j.nbd.2003.11.011

Heysieattalab, S., Naghdi, N., Zarrindast, M., Haghparast, A., Mehr, S., and Khoshbouei, H. (2016). The effects of GABAA and NMDA receptors in the shell-accumbens on spatial memory of METH-treated rats. Pharmacol. Biochem. Behav. 142, 23-35. doi: 10.1016/j.pbb.2015.12.008

Jayanthi, S., Deng, X., Noailles, P., Ladenheim, B., and Cadet, J. (2004). Methamphetamine induces neuronal apoptosis via cross-talks between endoplasmic reticulum and mitochondria-dependent death cascades. FASEB J. 18, 238-251. doi: 10.1096/fj.03-0295com

Kim, I., Xu, W., and Reed, J. (2008). Cell death and endoplasmic reticulum stress: disease relevance and therapeutic opportunities. Nat. Rev. Drug Discov. 7, 1013-1030. doi: $10.1038 / \mathrm{nrd} 2755$

Krasnova, I., and Cadet, J. (2009). Methamphetamine toxicity and messengers of death. Brain Res. Rev. 60, 379-407. doi: 10.1016/j.brainresrev.2009.03.002

Lebensztejn, D. (2000). Application of ursodeoxycholic acid (UDCA) in the therapy of liver and biliary duct diseases in children. Med. Sci. Monit. 6, 632-636.

Liu, F., Cui, Y., Ge, P., Luan, J., Zhou, X., and Han, J. (2015). Tauroursodeoxycholic acid attenuates inorganic phosphate-induced osteoblastic differentiation and mineralization in NIH3T3 fibroblasts by inhibiting the ER stress response PERK-eIF2alpha-ATF4 pathway. Drug Discov. Ther. 9, 38-44. doi: 10.5582/ddt. 2015.01008

Lynch, M. (2004). Long-term potentiation and memory. Physiological. Rev. 84, $87-136$.

Morgan, E., Woods, S., Poquette, A., Vigil, O., Heaton, R., and Grant, I. (2012), Visual memory in methamphetamine-dependent individuals: deficient strategic control of encoding and retrieval. Austr. N. Zeal. J. Psych. 46, 141-152. doi: $10.1177 / 0004867411433212$

Moszczynska, A., and Callan, S. (2017). Molecular, Behavioral, and Physiological Consequences of Methamphetamine Neurotoxicity: Implications for Treatment. J. Pharmacol. Exp. Ther. 362, 474-488. doi: 10.1124/jpet.116.238501

North, A., Swant, J., Salvatore, M., Gamble-George, J., Prins, P., Butler, B., et al. (2013). Chronic methamphetamine exposure produces a delayed, long-lasting memory deficit. Synapse 67, 245-257. doi: 10.1002/syn.21635

Oyadomari, S., and Mori, M. (2004). Roles of CHOP/GADD153 in endoplasmic reticulum stress. Cell Death Differ. 11, 381-389. doi: 10.1038/sj.cdd.4401373

Ozcan, U., Yilmaz, E., Ozcan, L., Furuhashi, M., Vaillancourt, E., Smith, R., et al. (2006). Chemical chaperones reduce ER stress and restore glucose homeostasis in a mouse model of type 2 diabetes. Science 313, 1137-1140. doi: 10.1126/ science. 1128294

Pastalkova, E., Serrano, P., Pinkhasova, D., Wallace, E., Fenton, A., and Sacktor, T. (2006). Storage of spatial information by the maintenance mechanism of LTP. Science 313, 1141-1144. doi: 10.1126/science.1128657

Psotta, L., Rockahr, C., Gruss, M., Kirches, E., Braun, K., Lessmann, V., et al. (2015). Impact of an additional chronic BDNF reduction on learning performance in an Alzheimer mouse model. Front. Behav. Neurosci. 9:58.

Sano, R., and Reed, J. C. (2013). ER stress-induced cell death mechanisms. Biochim. Biophys. Acta 1833, 3460-3470.

Scott, J. C., Woods, S. P., Matt, G. E., Meyer, R. A., Heaton, R. K., Atkinson, J. H., et al. (2007). Neurocognitive effects of methamphetamine: a critical review and meta-analysis. Neuropsychol. Rev. 17, 275-297. doi: 10.1007/s11065-0079031-0

Scoville, W. B., and Milner, B. (1957). Loss of recent memory after bilateral hippocampal lesions. J. Neurol. Neurosurg. Psychiatry 20, 11-21. doi: 10.1136/ jnnp.20.1.11

Shaerzadeh, F., Streit, W., Heysieattalab, S., and Khoshbouei, H. (2018). Methamphetamine neurotoxicity, microglia, and neuroinflammation. J. Neuroinflamm. 15:341.

Vorhees, C., and Williams, M. (2006). Morris water maze: procedures for assessing spatial and related forms of learning and memory. Nat. Protoc. 1, 848-858. doi: 10.1038/nprot.2006.116

Xu, B., Ye, Y., and Liao, L. (2019). Rapid and simple analysis of amphetaminetype illegal drugs using excitation-emission matrix fluorescence coupled with parallel factor analysis. Forensic. Sci. Res. 4, 179-187. doi: 10.1080/20961790. 2017.1349600

$\mathrm{Xu}, \mathrm{F}$., and Liu, L. (2019). Simultaneous determination of free methamphetamine, pethidine, ketamine and tramadol in urine by dispersive liquid-liquid microextraction combined with GC-MS. Forensic. Sci. Res. 4, 188-194. doi: $10.1080 / 20961790.2017 .1377386$

Yamamoto, B., Moszczynska, A., and Gudelsky, G. (2010). Amphetamine toxicities: classical and emerging mechanisms. Ann. N. Y. Acad. Sci. 1187, 101-121. doi: 10.1111/j.1749-6632.2009.05141.x

Yu, J., Huang, Y. W., and Chen, Z. (2003). [Improved alternative electro-stimulus Y-maze for evaluating the spatial memory of rats]. Zhejiang Da Xue Xue Bao Yi Xue Ban 32:140.

Yu, S., Zhu, L., Shen, Q., Bai, X., and Di, X. (2015). Recent advances in methamphetamine neurotoxicity mechanisms and its molecular pathophysiology. Behav. Neurol. 2015:103969.

Conflict of Interest: XW was employed by Becton, Dickinson and Company, Guangzhou, China.

The remaining authors declare that the research was conducted in the absence of any commercial or financial relationships that could be construed as a potential conflict of interest.

Copyright (c) 2021 Chen, Wei, Xu, Yu, Yong, Su and Tao. This is an open-access article distributed under the terms of the Creative Commons Attribution License (CC BY). The use, distribution or reproduction in other forums is permitted, provided the original author(s) and the copyright owner(s) are credited and that the original publication in this journal is cited, in accordance with accepted academic practice. No use, distribution or reproduction is permitted which does not comply with these terms. 